\title{
Determining the Electronic Confinement of a Sub-Surface Metallic State
}

\author{
Federico Mazzola, ${ }^{1}$ Mark T. Edmonds, ${ }^{2}$ Kristin Høydalsvik, ${ }^{1}$ \\ Damien J. Carter, ${ }^{3}$ Nigel A. Marks, ${ }^{3}$ Bruce C. C. Cowie, ${ }^{4}$ Lars \\ Thomsen, ${ }^{4}$ Jill A. Miwa, ${ }^{5}$ Michelle Y. Simmons, ${ }^{6}$ and Justin W. Wells ${ }^{1, *}$ \\ ${ }^{1}$ Department of Physics, Norwegian University of Science \\ and Technology (NTNU), N-7491 Trondheim, Norway \\ ${ }^{2}$ School of Physics, Monash University, Clayton, Victoria 3800, Australia \\ ${ }^{3}$ Nanochemistry Research Institute, Curtin University, PO Box U1987, Perth WA 6845 \\ ${ }^{4}$ Australian Synchrotron, 800 Blackburn Road, Clayton, Victoria 3168, Australia \\ ${ }^{5}$ Department of Physics and Astronomy, \\ Interdisciplinary Nanoscience Center (iNANO), \\ University of Aarhus, 8000 Aarhus C, Denmark \\ ${ }^{6}$ Centre of Excellence for Quantum Computation and Communication Technology, \\ School of Physics, University of New South Wales, Sydney, NSW 2052, Australia.
}

(Dated: September 20, 2014)

\begin{abstract}
Dopant profiles in semiconductors are important for understanding nanoscale electronics. Highly conductive and extremely confined phosphorus doping profiles in silicon, known as Si:P $\delta$-layers, are of particular interest for quantum computer applications, yet a quantitative measure of their electronic profile has been lacking. Using resonantly enhanced photoemission spectroscopy, we reveal the real-space breadth of the $\mathrm{Si}: \mathrm{P} \delta$-layer occupied states and gain a rare view into the nature of the confined orbitals. We find that the occupied valley-split states of the $\delta$-layer, the so-called $1 \Gamma$ and $2 \Gamma$, are exceptionally confined with an electronic profile of a mere 0.40 to $0.52 \mathrm{~nm}$ at full-width half-maximum; a result that is in excellent agreement with density functional theory calculations. Furthermore, the bulk-like $\mathrm{Si} 3 \mathrm{p}_{z}$ orbital from which the occupied states are derived, is sufficiently confined to lose most of its $\mathrm{p}_{z}$-like character, explaining the strikingly large valley splitting observed for the $1 \Gamma$ and $2 \Gamma$ states.
\end{abstract}

Keywords: $\mathrm{Si}: \mathrm{P}$ delta-layers, photoemission, 2D confinement, quantum computation 
Exceptionally sharp and high density doping profiles in semiconductors (known as $\delta$ layers) have been attracting interest over the last few years both because of their role as a platform for prototype quantum-computation components ${ }^{1-3}$ and for the fascinating insight into reduced dimension electronics. ${ }^{4-9}$ Shallow buried layers of phosphorus $(\mathrm{P})$ in bulk silicon ( $\mathrm{Si}$ ) are found to be a particularly suitable platform because of the high doping densities ${ }^{10}$ (and hence low sheet resistances ${ }^{11,12}$ ) and the almost atomically sharp confinement potentials which can be formed. ${ }^{13}$ Such physical confinement gives rise to a nearly-free electron-like occupied band dispersion, the calculation of which has been the centre of much effort. ${ }^{13-19}$ The occupied bandstructure has also recently been verified by photoemission spectroscopy (PES). ${ }^{6,20}$ The experimental verification is only possible because of a strongly enhanced photoemission intensity which occurs when an electron from a two dimensional (2D) initial state is photo-emitted via a well matched bulk-like final state. ${ }^{20,21}$

Whilst the growth and characterisation of Si:P $\delta$-layers has been widely studied using a range of techniques, important questions remain about the physical confinement of the 2D electronic states. The physical placement of the dopant atoms can be controlled and measured, ${ }^{10,11,22}$ but the electronic confinement, which is the key to understanding electronic parameters such as valley splitting, has been accessible only through calculations. ${ }^{6,13-19}$ Here we show that the strongly peaked photoemission enhancement which allows the $\delta$-layer states to be visible, also allows the physical profile of their wavefunction to be extracted. In particular, we demonstrate that not only can the breadth of the spectral envelope be investigated, but also the Bloch-like part of the wavefunction and the photoionisation crosssection. These parameters are found to be related to the bulk-like conduction band minimum $(\mathrm{CBM})$ from which the occupied $\delta$-layer states are derived and give a further insight into the nature of the $\delta$-layer electronic states and their confinement.

The presence of a high density, atomically sharp, $n$-type dopant layer in a bulk semiconductor causes the bulk CBM to become partially occupied in the region close to the dopant plane, thus creating a confined metallic layer (see Fig. 1 (a)). Density Functional Theory (DFT) and Angle Resolved Photoemission Spectroscopy (ARPES) have revealed that the two partially occupied states, referred to as $1 \Gamma$ (cyan) and $2 \Gamma$ (magenta), have a dispersion which is well approximated as parabolic, with the band minimum at the centre of the layer's 2D Brillouin zone (see Figs. 1 (b) \& (c), respectively). The measured valley splitting, or energy separation of $1 \Gamma$ and $2 \Gamma$, is large compared to the DFT calculated valley splitting 

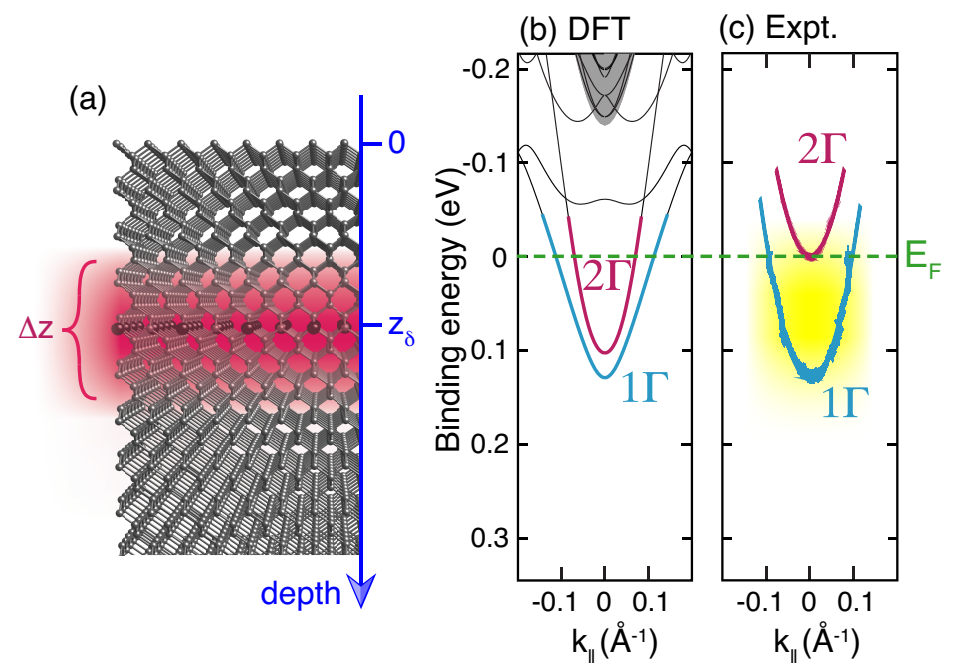

FIG. 1. $\delta$-layer bandstructure and density of states. (a) Idealised schematic of the $\delta$-layer sample where depth $z=0$ corresponds to the sample surface and $z=z_{\delta}$ the depth at which the dopants are placed. $\Delta z$ and the red shading indicate the breadth of the electrical confinement (dopant atoms are indicated as larger dark spheres). (b) DFT calculated bandstructure for an atomically sharp dopant plane (bulk CBM is shaded grey), and (c) the corresponding measured bandstructure (adapted from Ref. ${ }^{6}$ ). The yellow shading indicates the region which contributes to the angle integrated photoemission intensity in Fig. 2.

shown in Fig. 1 (b). However, the valley splitting in DFT calculations has been shown to be highly sensitive to the in-plane arrangement of phosphorus atoms in the $\delta$-plane (see Ref. ${ }^{13}$ and the Supplementary Material of Ref. ${ }^{6}$ ), the details of which have not been observable experimentally. In all other aspects than the valley splitting, the agreement between DFT and measurements is excellent.

\section{RESULTS}

A photoemission experiment performed around normal emission, with a suitable angular integration $\left( \pm 1.7^{\circ}\right)$ can be taken as a measure of the occupied density of states (DOS). The DOS integrated in this range consists of contributions from both $1 \Gamma$ and $2 \Gamma$ (see Fig. 1 (c)). Photoemission is also sensitive to the available unoccupied states; in order to transport the excited electron from the buried $\delta$-layer to the surface, the final state must be delocalised from the $\delta$-layer - in other words it is a 3D bulk-like state. Since both energy and momen- 
tum are conserved in the excitation, ${ }^{23}$ the photoemission probability depends strongly on the availability of a final state of suitable energy and momentum. Probing this condition ultimately allows us to measure the real-space profile of the $2 \mathrm{D}$ initial state.

Since the photo-excitation conserves energy, varying the excitation energy changes the availability of final states. This is seen in the experimental data (Fig. 2 (a)) as a change in the photoemission intensity near the Fermi level. Using the relationship $k_{\perp} \approx \sqrt{\left(2 m / \hbar^{2}\right)\left(V_{0}+h \nu-E_{b}-\Phi\right)}$, where $V_{0}$ is the inner potential and $\Phi$ is the sample workfunction, ${ }^{23}$ the photon energy scale can be converted simply into units of crystal momentum (Fig. 2 (b)). Integrating the binding energy region -0.1 to $+0.1 \mathrm{eV}$ (i.e. the total intensity from the $\delta$-layer initial states; $1 \Gamma$ and $2 \Gamma$ ) makes the resonant behaviour more clear; the integrated intensity from Fig. 2 (b) is plotted in Fig. 2 (d). The photoemission intensity can generally be described as a function which is periodic in momentum, with a characteristic width in $k_{\perp}$. A synchrotron beamline with a lower photon energy range allows an additional period at lower $k_{\perp}$ to be observed (Fig. 2 (c) $)^{24}$.

Due to the 3D nature of the bulk-like final state $\left(b_{f}\right)$, the final state will show a periodic dispersion in energy as a function of momentum $k_{\perp} \cdot{ }^{21,23,25}$ The $2 \mathrm{D}$ nature of the initial states $1 \Gamma$ and $2 \Gamma$ results in a lack of such dispersion, thus they are depicted at constant binding energy (see Fig. 3 (a)). Since the photo-excitation involves a well defined amount of energy, and a negligible amount of $k_{\perp},{ }^{23}$ it is naïvely possible to see where the photo-excitation can occur. At two different photon energies chosen for illustration, $h \nu_{1}$ and $h \nu_{2}$, excitation from $2 \Gamma$ to the bulk state can only occur for the particular values of $k_{\perp}$ indicated in Fig. 3 (a). In fact, it is this consideration (in combination with the assumption of a free-electron-like vacuum state) which allows the experimental data (Fig. 2 (b-d)) to be plotted in units of $k_{\perp} \cdot{ }^{23}$ 

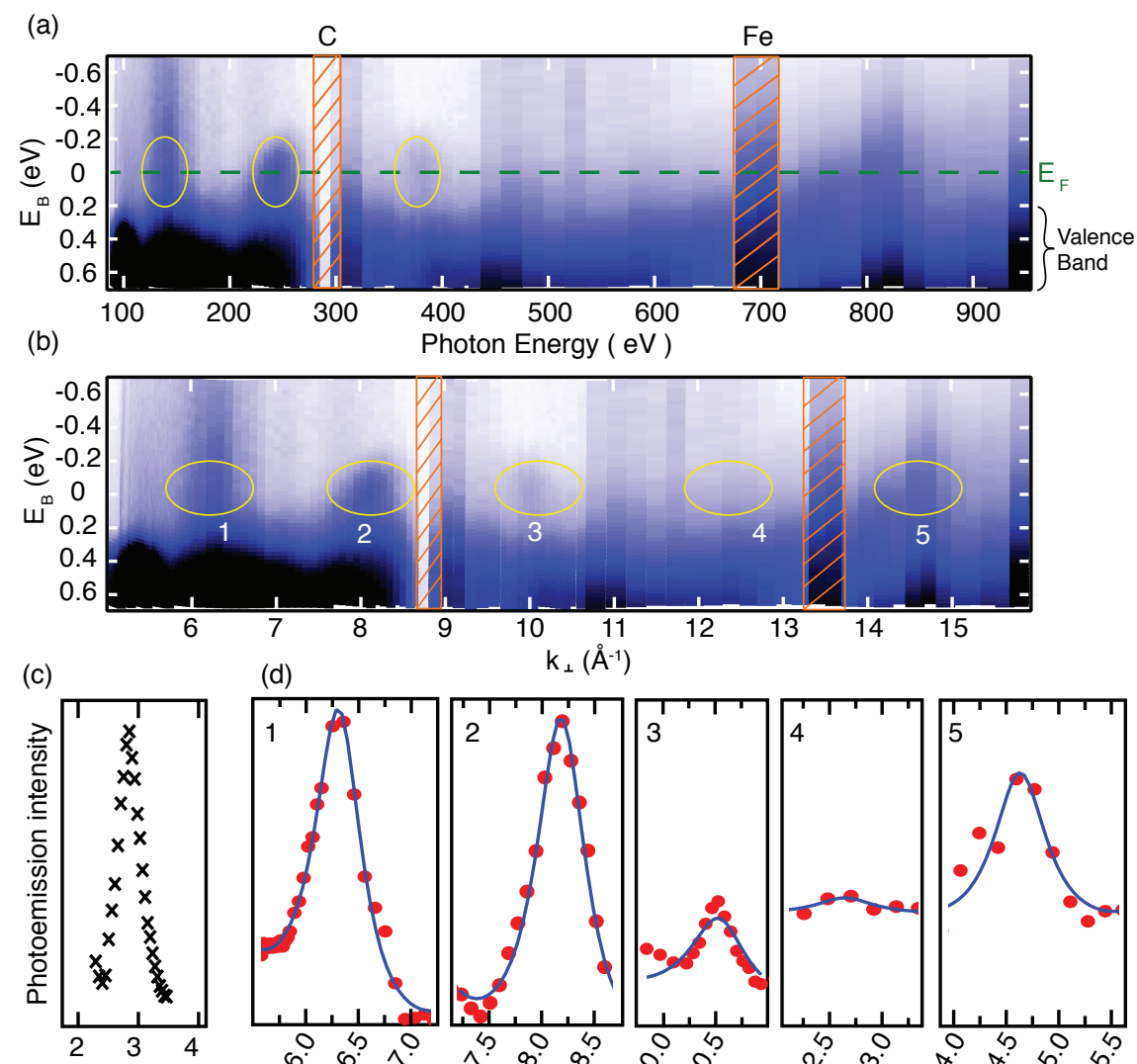

(d)
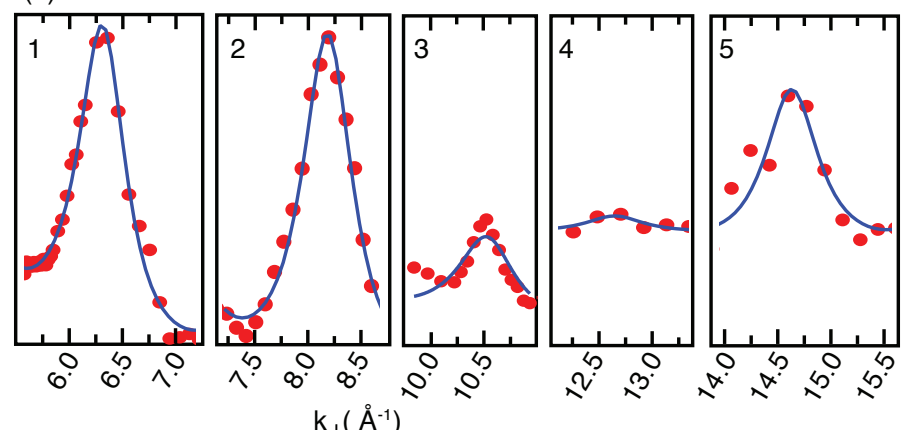

$k_{\perp}\left(\AA^{-1}\right)$

FIG. 2. Photoemission measurements of the $\delta$-layer states. (a) Photoemission intensity around the Fermi level for a range of photon energies (darker shades indicate higher intensity). The Fermi level $\left(E_{F}\right)$ and the approximate energy of the valence band is indicated. The intensity has been normalised to the beamline flux. The markers ' $\mathrm{C}$ ' and ' $\mathrm{Fe}$ ' indicate where normalisation is hindered by strong absorption by the beamline optics. Within the band gap, the photoemission intensity is enhanced at particular photon energies (enclosed in yellow ellipses). (b) The same photoemission intensity, converted into units of $k_{\perp}$ with five enhancements indicated by yellow ellipses. (c) Integrated photoemission intensity for a similar sample, at smaller $k_{\perp}$ (the absolute intensity cannot be compared between panels). (d) 1-5: Photoemission intensity (integrated in the energy range -0.1 to $+0.1 \mathrm{eV}$ ) for the range of $k_{\perp}$ corresponding to each enhancement (as numbered in panel b). The blue line is a curve of form $I \propto(\chi-1)^{2} /\left(1+\chi^{2}-2 \chi \cos \left(k_{\perp} a-\pi\right)\right)$ (see main text for details). 

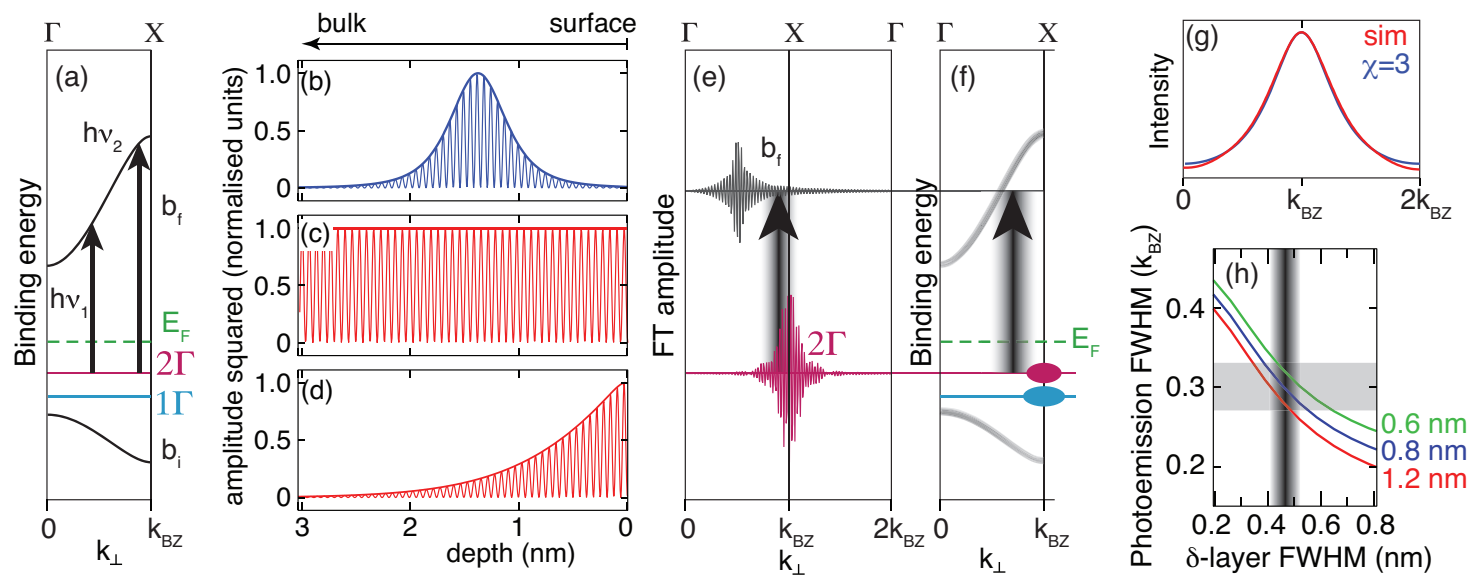

FIG. 3. Schematic of the photoemission process. (a) Photoemission between a 2D initial state $(2 \Gamma)$ and a bulk-like final state $\left(b_{f}\right)$. In the simple picture, for specific photon energies $\left(h \nu_{1}\right.$ and $\left.h \nu_{2}\right)$ the photo-excitation can only occur at particular values of $k_{\perp}$ such that energy and momentum are conserved. (b) initial state and (c) final state models; the states are described by Lorentzian and semi-infinite distributions, respectively, but also comprise a Bloch-like part. The initial state $(1 \Gamma$ or $2 \Gamma$ ) is centred around the dopant plane $1.4 \mathrm{~nm}$ beneath the surface. (d) The same final state with an exponential decay describing the accessibility to photoemission by including a photoelectron MFP. (e) Fourier transform of initial and final states, showing that they have spectral weight over a range of $k_{\perp}$. The broad black arrow shows that photo-excitation can occur, even though the centre values of $k_{\perp}$ are mismatched. (f) The same photo-excitation in the schematic bandstructure. Although $2 \Gamma$ is non-dispersive, the spectral weight is strongly enhanced at particular $k_{\perp}$ (indicated by the magenta ellipse). The final state is dispersive with a non-zero width in $k_{\perp}$. (g) The intensity of the convolution of the initial and final states, showing a strongly peaked distribution. Our numerical simulation (red) together with the expression from Ref. $^{21}$, with $\chi=3$. In this particular case, a photoelectron MFP of $1 \mathrm{~nm}$, and an initial state FWHM of $0.3 \mathrm{~nm}$ are used. (h) Dependence of the FWHM of the simulated photoemission intensity on the initial state FWHM and photoelectron MFP. The horizontal grey band indicates the experimentally determined $\mathrm{FWHM}=0.3 k_{B Z} \pm 10 \%$ and the vertical grey band indicates the corresponding range of initial state FWHM.

\section{DISCUSSION}

Although the initial states are dispersionless in $k_{\perp}$, they are not of uniform intensity. This can be seen from a Fourier transform (FT) of the real-space distribution, which reveals that 
the distribution in $k_{\perp}$ has a width defined by the inverse of the real-space breadth, centred on the Bloch momentum (Fig. $3(\mathrm{e})$ ). $1 \Gamma$ and $2 \Gamma$ are derived from the bulk CBM close to the out-of-plane Brillouin Zone boundary, hence they have Bloch momentum similar to that of the BZ boundary, $k_{B Z} \cdot{ }^{20}$ The strongly peaked FT intensity is the origin of the strongly peaked photoemission intensity; at particular photon energies, photo-excitation can occur at values of $k_{\perp}$ which match the initial state Bloch momentum where the majority of the initial state spectral weight is found, with a suitable final state.

In fact, the situation is not quite so simple since the final state breadth must also be considered. Since the final state is bulk-like, it can be depicted as a Bloch-like oscillation extending infinitely into the bulk (Fig. 3 (c)). However, the final state probed by photoemission is attenuated into the bulk by an exponential decay, and the exponent depends on the mean-free-path (MFP) of the photoelectron, which in turn depends on the kinetic energy. Fig. 3 (d) depicts a final state modulated by a realistic MFP. The FT of this final state now has a non-zero width in $k_{\perp}$ (see Fig. $3(\mathrm{e})$ ).

The implication of the finite FT widths is that the simplified picture of photoemission presented in Fig. 3 (a) needs to be modified; photo-excitation can occur at values of $k_{\perp}$ which are not well matched to a final state, but the probability is reduced. This is illustrated in Fig. 3 (f), where the photo-excitation is depicted by the broad arrow terminating at the righthand side of the final state whilst originating from the lefthand side of the initial state. By comparison with the FT amplitudes presented in Fig. 3 (e), it can be seen that such a photo-excitation can occur since both the initial and final states have a non-negligable intensity here. In other words, the photoemission probability at a particular photon energy depends on the overlap of the initial state and final state FTs. As the photon energy is varied, there will be values at which the overlap is maximised, thus accounting for the peaked photoemission intensity observed in the measurements. The peak shape is determined by the convolution of the initial and final states (Fig. $3(\mathrm{~g})$ ). More specifically, the FWHM of the peaked photoemission intensity depends on the real-space breadth of the initial state, and the MFP assumed for the final state. The FWHM of the photoemission intensity is evaluated for a realistic range of MFP (0.6 to $1.2 \mathrm{~nm}$ for the energy range 150 to $1000 \mathrm{eV}^{26}$ and for a realistic range of initial state breadths (Fig. $3(\mathrm{~h})$ ).

The simulation presented above focusses on the novel case of a sub-surface 2D dopant plane. However, photoemission from other 2D initial states (in particular, surface states) is 
(a)

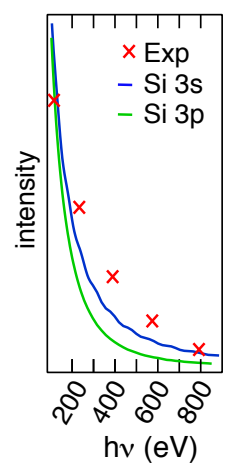

(b)

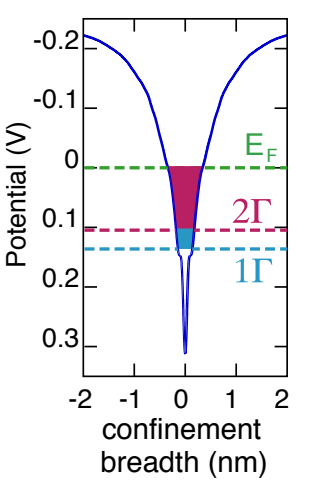

(c)

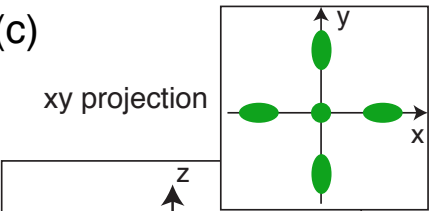

FIG. 4. (a) Calculated Si 3s (blue) and 3p (green) photoionisation crosssections (adapted from Ref. ${ }^{29}$ ) together with the experimental photoemission intensity (corrected for beamline flux and integration time). (b) The DFT calculated confinement potential, with the experimentally determined Fermi level and the approximate energies of the $1 \Gamma$ and $2 \Gamma$ band minima indicated. The breadth of the confinement potential at these energies is $0.68 \mathrm{~nm}\left(E_{F}\right), 0.36 \mathrm{~nm}(2 \Gamma)$ and $0.30 \mathrm{~nm}$ (1Г). (c) A schematic of the confined $3 \mathrm{p}$ orbitals, showing the s-like shape of the confined $\mathrm{p}_{z}$ orbital.

well understood, and a peaked photoemission intensity also occurs. ${ }^{21,25}$ This peaked intensity has been described by $I \propto(\chi-1)^{2} /\left(1+\chi^{2}-2 \chi \cos \left(k_{\perp} a-\pi\right)\right)$, where $I$ is the photoemission intensity, $a$ is the reciprocal unit cell and $\chi$ is a parameter describing the bandwidth of the $2 \mathrm{D}$ orbital relative to the bulk and the self-energy of the surface state (see Ref. ${ }^{21}$ for full description). In order to facilitate a comparison between our simulation involving a sub-surface 2D initial state and earlier work on surface localised states, a curve of this form has been overlaid on both our numerical simulation presented in Fig. 3 (g), and the data in Fig. 2(d). In our case $\chi$ is unknown, but best agreement with our simulation is found when $\chi \approx 3$ (c.f. $\chi=1.6$ and 1.8 for the two surface states considered in Ref. ${ }^{21}$ ). In other words, although our initial state has quite another origin than the surface states considered in earlier works, the photoemission model is very similar.

The FWHM of the measured photoemission intensity peaks has been found to be $\approx$ $0.9 \AA^{-1}$, equivalent to $\approx 0.3 k_{B Z}$, with an uncertainty of $\approx 10 \%$. Our simulations show that this corresponds to a real-space FWHM of the initial state of just 0.40 to $0.52 \mathrm{~nm}$ (assuming an MFP in the range 0.6 to $1.2 \mathrm{~nm}$ ). Although the estimate for the initial state's FWHM depends on the estimate of the MFP, in this range the dependence is weak; as seen in Fig. 
3(h). This initial state breadth is exceptionally sharp, especially given that the distribution of dopant atoms is thought to be somewhat broader than this. ${ }^{11}$ However, the same DFT calculation used to generate the bandstructure in Fig. 1 (b) yields a confinement potential on this length scale (Fig. 4 (b)). By aligning the DFT energy scale with the experimentally determined Fermi level, the confinement breadth at particular energies can be estimated. The breadth of the confinement potential is $0.68 \mathrm{~nm}$ (at $E_{F}$ ), $0.36 \mathrm{~nm}$ (at the $2 \Gamma$ band minimum) and $0.30 \mathrm{~nm}$ (at the $1 \Gamma$ band minimum) ${ }^{27}$.

As well as periodic oscillations in the photoemission intensity, the peak intensity rapidly diminishes with increasing $k_{\perp}{ }^{28}$. This is due to a decreasing photoionisation cross-section and is a common observation which is well understood. ${ }^{29}$ Since the $1 \Gamma$ and $2 \Gamma$ states are primarily derived from bulk Si $3 \mathrm{p}_{z}$ orbitals, one could expect that their photoionisation cross-section follows the calculated Si 3p values (see Fig. 4 (a)). However, the observed decay is weaker than the calculated curve, and is more similar to (but still weaker than) the calculated Si 3s cross-section. This is another consequence of the strong confinement; in the $\delta$-layer, the $\mathrm{p}_{z}$ orbital is so strongly confined that it is more akin to a small, nearly-spherical s-orbital (see Fig. 4 (c)). Thus, the weakly decaying cross-section is indicative of an orbital which is very small compared to a bulk-like Si $3 \mathrm{p}_{z}$.

It is intriguing that such a sharp $\delta$-layer confinement can exist, when the distribution of dopant atoms is known to be broader. ${ }^{11}$ Since the photoemission experiment is giving information on the initial states only when they are occupied, the relevant breadth corresponds to a metallic dopant density, in other words, it is the breadth at the 'tip' of the dopant distribution which is relevant, rather than its FWHM. By comparison with Ref. ${ }^{11}$, it can be inferred that phosphorus densities in the order of $\approx 5 \times 10^{20} \mathrm{~cm}^{-3}$ exist over a similar breadth (i.e. 0.40 to $0.52 \mathrm{~nm}$ ), thus it appears that dopant densities of this magnitude are needed to create a metallic 2D layer - this doping density is significantly higher than in a $3 \mathrm{D}$ degenerate semiconductor, presumably because not all the donated carriers remain in the $2 \mathrm{D}$ metallic region, but also contribute to the strong (but not metallic) $n$-type doping of the surrounding silicon.

The out-of-plane confinement of the $\delta$-layer states has wider implications for complex dopant arrangements; for example, the fact that we observe a confinement FWHM equivalent to just a few atomic spacings brings with it the implication that multiple $\delta$-layers could be treated as independent if their separation is much larger than this confinement length scale. 
Conversely, multiple stacked $\delta$-layers with a separation comparable to this length scale would be expected to interact. This view is supported by recent calculations, ${ }^{17,30}$ and forms the basis of novel 3-D device architectures based on stacked $\delta$-layers. ${ }^{31}$ Although our work has focussed on the out-of-plane confinement of uniform 2D dopant structures, it is reasonable to assume that a similar length scale defines the in-plane confinement of patterned dopant structures, such as 1-D wires ${ }^{7}$ and $0-\mathrm{D}$ quantum $\operatorname{dots}^{1}$ and interacting multiple quantum dot devices. ${ }^{2,3}$

\section{CONCLUSION}

The combination of synchrotron photoemission, simulations and DFT calculations provides a detailed insight into the confinement of the $\mathrm{Si} 3 \mathrm{p}_{z}$ derived states $(1 \Gamma$ and $2 \Gamma)$ which are responsible for the metallic 2D properties of a Si:P $\delta$-layer. ${ }^{6,20}$ Strongly peaked photoemission intensity is a consequence of a strongly confined initial state, and from the peak width it is possible to reconstruct the real-space breadth. Excellent agreement is found when the initial state is modelled as a Lorentzian envelope of FWHM 0.40 to $0.52 \mathrm{~nm}$, containing a Bloch-like wavefunction similar to that of the bulk CBM from which the $1 \Gamma$ and $2 \Gamma$ states are derived. Such strong confinement results in the cross-section of the orbital being modified away from that of an unconfined Si 3p orbital, as well as contributing to an unusually large valley splitting. By comparison with measurements of the phosphorus distribution, it appears that the confinement is derived from the high density 'tip' of the dopant distribution; indicating that the formation of a 2D metallic layer requires a particularly high dopant density of around $5 \times 10^{20} \mathrm{~cm}^{-3}$. DFT calculations are in good agreement, not only in reproducing the band-structure, but also in producing a confinement potential which is consistent with the experimental observations. Importantly, the approach described here is not limited to Si:P, but is applicable to understanding any 2D metallic layer which can be observed by photoemission. Furthermore, these results should provide valuable insight into the robustness of such doping profiles and their bearing on nanoscale electronics.

Experimental details: $\delta$-doped $\operatorname{Si}(001)$ samples are made in situ by first preparing a clean $\mathrm{Si}(001)$ sample (thermal annealing up to $\approx 1520 \mathrm{~K}$ in a base pressure of $\approx 2 \times 10^{-10} \mathrm{mbar}$ ), and a sharp Si $2 \times 1$ reconstruction is seen by low energy electron 
diffraction (LEED). This is followed by deposition of $\approx 1 / 4$ of a monolayer of phosphorus dopants (from phosphine gas dosed at room temperature for 5 minutes at a pressure of $5 \times 10^{-9}$ mbar) and subsequently incorporated into the surface by annealing to $520 \mathrm{~K}$ (as described elsewhere $\left.{ }^{10,11,22}\right)$. An $\approx 1 \mathrm{~nm}$ thick silicon epilayer is formed atop the dopant layer, using a thermal evaporator, and the coverage is monitored and estimated by the relative intensities of the $\mathrm{Si}$ and $\mathrm{P}$ core levels acquired during the experiment. Such a coverage is selected to ensure that a confinement potential is formed which remains accessible by photoemission. ${ }^{6,20}$ Core-level spectroscopy measurements confirmed minimal phosphorus dopant segregation for all anneal steps during sample preparation.

The data were collected at two beamlines, the Soft-X-Ray beamline (SXR) at the Australian synchrotron (Melbourne) ${ }^{32}$ and at I4 beamline in MAX-IV laboratory (Lund, Sweden). ${ }^{33}$ The measurements were performed at room temperature and the angular acceptance chosen to be approximately the same in both cases $\left( \pm 1.7^{\circ}\right.$ centred at normal emission).

The data presented here have undergone an intensity normalization to the beamline flux and the binding energy has been calibrated using a gold Fermi level.

DFT methods section: The $1 \Gamma$ and $2 \Gamma$ states (Fig. 1(b)) have been calculated using the SIESTA code ${ }^{34}$ and the methods described by Carter et al. in Ref. ${ }^{17}$. An elongated three-dimensional unit cell, a Double-Numerical-plus Polarization (DNP) atom centered basis set, and the Generalized-Gradient Approximation (GGA-PBE) ${ }^{35}$ were used in order to represent the $\delta$-layer. A suitable degree of electronic separation in the direction normal to the dopant plane, is guaranteed by the separation of 40 atomic layers between the $\delta$-layer and its periodic images. In the direction parallel to the dopant plane, a $4 \times 4$ simulation cell of 16 atoms has been adopted (in particular, $4 \mathrm{P}$ and $12 \mathrm{Si}$ in the $\delta$-layer, the same as the experimental concentration of $1 / 4 \mathrm{ML}$, or $\left.2.2 \times 10^{14} \mathrm{~cm}^{-210}\right)$. The resulting band structure, showing the $1 \Gamma$ and $2 \Gamma$ states is represented in Fig. $1(\mathrm{~b})$.

Acknowledgements: We thank J. Adell for technical support at the MAX-IV laboratory and O. Warschkow for assisting with the measurements and DFT calculations. J.A.M acknowledges the Lundbeck Foundation via L. Hornekær for providing financial support. M.T.E. is supported by the ARC Laureate Fellowship project of M.S. Fuhrer. M.Y.S. is supported by the Australian Research Council (ARC) Centre of Excellence for Quantum Computation and Communication Technology (project number CE110001027) and M.Y.S. 
is also supported by the US National Security Agency and the US Army Research Office under contract number W911NF-08-1-0527. M.Y.S. and N.A.M. acknowledge the ARC for providing fellowships. J.W.W. acknowledges NTNU's TSO materialer program for supporting the collaboration. This research was partially undertaken on the Soft X-Ray beamline at the Australian Synchrotron, Victoria, Australia.

* quantum.wells@gmail.com

[1] Fuechsle, M; Miwa, J.A.; Mahapatra, S; Ryu, H; Lee, S; Warschkow, O; Hollenberg, LCL; Klimeck, G; Simmons, M.Y. A Single-Atom Transistor, Nat. Nano. 2012, 7, 242-246.

[2] Weber, B; Tan, Y.H.M; Mahapatra, S; Watson, T.F; Ryu, H; Rahman, R; Hollenberg, L.C.L; Klimeck, G; Simmons, M.Y. Spin Blockade and Exchange in Coulomb-Confined Silicon Double Quantum Dots. Nat Nano, 2014, 9, 430435.

[3] Watson, T.F; Weber, B; Miwa, J.A; Mahapatra, S; Heijnen, R.M.P; Simmons, M.Y. Transport in Asymmetrically Coupled Donor-Based Silicon Triple Quantum Dots. Nano Letters 2014, $14,1830-1835$.

[4] Polley, C.M; Clarke, W.R; Miwa, J.A; Simmons, M.Y; Wells, J.W. Microscopic Four-PointProbe Resistivity Measurements of Shallow, High Density Doping Layers in Silicon. Appl. Phys. Lett. 2012, 101, 262105.

[5] Ohno, K; Heremans, F.J; Bassett, L.C; Myers, B.A; Toyli, D.M; Jayich, A.C.B; Palmstrøm, C.J; D. D. Awschalom, D.D. Engineering Shallow Spins in Diamond with Nitrogen DeltaDoping. Appl. Phys. Lett. 2012, 101, 082413.

[6] Miwa, J.S; Warschkow, O; Carter, D.J; Marks, N.A; Mazzola, F; Simmons M.Y. and J. W. Wells. Valley Splitting in a Silicon Quantum Device Platform. Nano Letters 2014, 14, 15151519.

[7] Weber, B; Mahapatra, S; Ryu, H; Lee, S; Fuhrer, A; Reusch,T.C.G; Thompson, D.L; Lee, W.C.T; Klimeck, G; Hollenberg, L.C.L; Simmons, M.Y. Ohm's Law Survives to the Atomic Scale. Science 2012, 335, 64-67.

[8] Drumm, D.W; Smith, J.S; Per, M.C; Budi, A; Hollenberg, L.C.L; Russo, S.P. Ab Initio Electronic Properties of Monolayer Phosphorus Nanowires in Silicon. Phys. Rev. Lett. 2013, 110, 126802 . 
[9] Ryu, H; Lee, S; Weber, B; Mahapatra, S; Hollenberg, L; Simmons, M; Klimeck, G. Atomistic Modeling of Metallic Nanowires in Silicon, Nanoscale 2013, 5 8666-8674.

[10] McKibbin, S.R; Clarke, W.R; Fuhrer, A; Simmons, M.Y. Optimizing Dopant Activation In Si:P Double $\delta$ Layers. J. Cryst. Growth 2010, 312 3247-3250.

[11] Polley, C.M; Clarke, W.R; Miwa, J.A; Scappucci, G; Wells, J.W; Jaeger, D.L; Bischof, M.R; Reidy, R.F; Gorman, B.P; Simmons, M. Exploring the Limits of n-type Ultra-Shallow Junction Formation. ACS Nano 2013, 7, 5499-5505.

[12] Mazzola, F; Polley, C.M; Miwa, J.A; Simmons, M.Y; Wells, J.W. Disentangling Phonon and Impurity Interactions in $\delta$-Doped $\operatorname{Si}(001)$. Appl. Phys. Lett. 2014, 104, 173108.

[13] Carter, D.J. Marks, N.A; Warschkow, O; McKenzie, D.R. Phosphorus $\delta$-Doped Silicon: MixedAtom Pseudopotentials and Dopant Disorder Effects.' Nanotechnology 2011, 22, 065701.

[14] Carter, D.J; Warschkow, O; Marks, N.A; McKenzie, D.R. Electronic Structure Models of Phosphorus $\delta$-Doped Silicon. Phys. Rev. B 2009, 79, 033204 and 200980049901.

[15] Lee, S; Ryu, H; Campbell, H; Hollenberg, L.C.L; Simmons, M.Y; Klimeck, G. Electronic Structure of Realistically Extended Atomistically Resolved Disordered Si:P $\delta$-Doped Layers. Phys. Rev. B 2011, 84, 205309.

[16] Drumm, D.W; Hollenberg, L.C.L; Simmons, M.Y; Friesen, M. Effective Mass Theory of Monolayer $\delta$-Doping in the High-Density Limit. Phys. Rev. B 2012, 85, 155419.

[17] Carter, D.J; Warschkow, O; Marks, N.A; McKenzie, D.R. Electronic Structure of Two Interacting Phosphorus $\delta$-Doped Layers in Silicon. Phys. Rev. B 2013, 87, 045204.

[18] Drumm, D.W; Budi; A; Per, M.C; Russo, S.P; Hollenberg, L.C.L. Ab initio Calculation of Valley Splitting in Monolayer $\delta$-Doped Phosphorus in Silicon. Nanoscale Res. Lett. 2013, 8, 111.

[19] Smith, J.S; Cole, J.H; Russo, S.P. Electronic Properties of $\delta$-Doped Si:P and Ge:P Layers in the High-Density Limit Using a Thomas-Fermi Method. Phys. Rev. B 2014, 89, 035306.

[20] Miwa, J.A; Hofmann, P; Simmons, M.Y; Wells, J.W. Direct Measurement of the Band Structure of a Buried Two-Dimensional Electron Gas. Phys. Rev. Lett. 2013, 110, 136801.

[21] Louie, S.G; Thiry, P; Pinchaux, R; Petroff, Y; Chanderis, D; Lecante, J. Periodic Oscillations of the Frequency-Dependent Photoelectric Cross Sections of Surface States: Theory and Experiment. Phys. Rev. Lett. 1980, 44, 549-553.

[22] Goh, K.E.J; Simmons, M.Y. Impact of Si Growth Rate on Coherent Electron Transport in 
Si:P Delta-Doped Devices. Appl. Phys. Lett. 2009, 95, 142104.

[23] Himpsel, F.J. Experimental Determination of Bulk Energy Band Dispersions. Appl. Opt. 1980, 19, 3964-3970.

[24] Since the data in Figs. 2 (c) and (d) where collected on two different beamlines, a direct comparison of the absolute intensity has not been possible.

[25] Hofmann, P; Søndergaard, C; Agergaard, S; Hoffmann, S.V; Gayone, J.E; Zampieri, G; Lizzit, S; Baraldi, A. Unexpected Surface Sensitivity at High Energies in Angle-Resolved Photoemission. Phys. Rev. B 2002, 66, 245422.

[26] Seah, M.P; Dench, W.A. Quantitative Electron Spectroscopy of Surfaces: A Standard Data Base for Electron Inelastic Mean Free Paths in Solids. Surf. Interface Anal. 1979, 1, 2-11.

[27] Note that both $1 \Gamma$ and $2 \Gamma$ contribute to the photoemission intensity.

[28] A precise normalisation of the intensity of the peak marked ' 5 ' in Fig. 2 (b) is difficult.

[29] Yeh, J.J; Lindau, I. Atomic Subshell Photoinoization Cross Sections and Asymmetry Parameters: $1 \leq Z \leq 103$. At. Data Nucl. Data Tables 1985, 32, 1-155.

[30] Drumm, D; Per, M; Budi, A; Hollenberg, L; Russo,S. Ab Initio Electronic Properties of Dual Phosphorus Monolayers in Silicon. Nanoscale Res. Lett. 2014, 9, 443.

[31] Scappucci, G; Capellini, G; Klesse, W.M; Simmons, M.Y. New Avenues to an Old Material: Controlled Nanoscale Doping of Germanium. Nanoscale 2013, 5, 2600-2615.

[32] Cowie, B.C.C; Tadich, A; Thomsen, L. The Current Performance of the Wide Range (90-2500 eV) Soft X-ray Beamline at the Australian Synchrotron. AIP Conference Proceedings 2010, 1234, 307-310.

[33] Jensen, B; Butorin, S; Kaurila, T; Nyholm, R; Johansson, L. Design and Performance of a Spherical Grating Monochromator Used at MAX. Nuclear Inst. and Methods in Physics Research, A 1997, 394, 243-250.

[34] Soler, J.M; Artacho, E; Gale, J.D; Garcïa, A; Junquera, J; Ordejón, P; Sánchez-Portal, D. The Siesta Method for ab initio Order-N Materials Simulation," J. Phys.: Cond. Matt. 2002. 14,2745 .

[35] Perdew, J.P; Burke, K; Ernzerhof, M. Generalized Gradient Approximation Made Simple. Phys. Rev. Lett. 1996, 77, 3865-3868. 